\title{
Investigating the Impact of Road Condition Complexity on Driving Workload Based on Subjective Measurement using NASA TLX
}

\author{
Sugiono Sugiono ${ }^{1, a}$, Denny Widhayanuriyawan ${ }^{2}$, and Debrina P. Andriani ${ }^{1}$ \\ ${ }_{1}^{1}$ Industrial Engineering Dept., Universitas Brawijaya (UB), Indonesia \\ ${ }^{2}$ Mechanical Engineering Dept., Universitas Brawijaya (UB), Indonesia
}

\begin{abstract}
Prior researchers indicate that mental load is one of the most important contributors to a traffic accident. The aim of the paper is to investigate the impact and the correlation of road condition and driving experience on driver's mental workload. The driving test consists of 3 road complicity situation (urban road, highway, rural road) with 26 drivers with average 21 years old in different experience level (average 4.08 years' experience). NASA TLX questioner is used as subjective driver's mental load measurement with three dimensions relate to the demands imposed on the subject (Mental, Physical and Temporal Demands) and three to the interaction of a subject with the task (Effort, Frustration, and Performance). There are 3 cameras placed on the left side, right side and front car to identify the road condition. According to experiment, it was found that drivers felt that frustration level, business, and mental-demand factors dominate the impact on high-level workload $(96.15 \%)$. Highway road conditions provide an average overall workload score of 62 (OWS) which was better compared to city road $(\mathrm{OWS}=69)$ and rural road (OWS $=66)$. Based on street complexity, it is necessary to improve road conditions that resemble highway road by reducing potential hazard.
\end{abstract}

Keywords: Mental driving workload, NASA TLX, Overall workload score (OWS)

\section{Introduction}

Driving is main human activity which is conducted to support daily activities and mobility in the shortest time possible. This initiated large government investment in providing safe and convenient transportation system. The driving complexity causes road accidents, not only due to physical factors such as cars and road conditions but also due to human factors themselves such as human error and excessive stress levels. Fastenmeir and Gstalter [1] research define that the complexity of driving depends on elements such as road design (highway, rural road, city road), road layout (straight, curved, inclined, junction) and traffic flow (high density). The study of the situation and the pattern of the relationship between drivers with cars and driving environments in order to reduce or avoid possible risk. The driver's ability to meet driving needs becomes an important capital in realizing safety driving [2]. The ability to adapt quickly, such as reducing speed, changing the direction of the vehicle is very much dependent on driving experience and mental condition (load) of the driver.

Schlegel [3] defines driving workload as the total amount of resources or driver's ability to achieve a driving task. Further driving workload reflects the driving need to achieve performance tasks that depend on individual drivers and skills. Driving workload depends on the task (demand, complexity, and difficulties), drivers (age, fatigue, abilities, experience, etc.) and environment (dynamic/changing, familiar, etc.). Total driving workload consists of every aspect relating to driving task and nondriving task [4]. Driving tasks consist of street monitors making lateral/longitudinal action by using main control (e.g. steering and pedals) and manipulating other controls related to driving safety (e.g. windshield) and displays. Non-driving tasks such as operating the secondary interface (e.g. AC, audio/video, reading maps, phone, drink, etc.). Both of the above driving tasks require the mental and physical actions. Mental actions include acceptance, information processing and decision making involving human five senses and memory systems. Physical actions involve providing energy to move human organs such as hands, feet, head, and eyes to drive. Driving situations have a major impact on the mental workload manifested in the relationship pattern between task demand and driving performance [5]. The complexity of the environment strongly affects drivers to respond and produce good performances, for example when driving around the market or urban streets with a huge number of passersby, the driver is required to immediately process all the information appropriately. Events like this can cause overload to keep driving performance. Therefore takes the correct strategy and qualified physical condition.

An understanding of mental workload and physical workload on drivers is essential to building a powerful Driving alert system. Shih Nan Lu et al. explains that the Industrial Technology Research Institute (ITRE) has also used the ultra-wide bandwidth (UWB) technique to integrate low power pulsed electromagnetic (EM) waves to precisely measure the driver's physiological signals such as his heartbeat, respiration, etc. [6]. ITRE has developed a driving safety system focusing not only on the physical technology of the vehicle but also considering the human. Furthermore, the project developed will create a driving safety system that involves a mental load that will be measured in a subjective offline with National Aeronautics and Space Administration Task

\footnotetext{
${ }^{a}$ Corresponding author: sugiono_ub@ub.ac.id
} 
Load Index (NASA TLX) and objective (heart rate, EEG). A driving safety system created will record the initial measurement values of driving and analyse other information (bedtime, physical complaints experienced) to establish the maximum allowable minimum and allowable limits. At the time of driving, if the security system receives the insert value exceeds the maximum limit then the alerting system will provide the mark in the form of sound and visual through the mobile phone application. Therefore the driver will adjust their driving which ultimately reduces the risk of accidents. As an initial stage of research, this paper will exhibit the measurement of subjective mental burden using NASA TLX on the diverse trajectory (market/city road, highway, rural road) and diverse driving experiences.

\section{Material and Methodology}

Basically, human activity can be differentiated in physical work (muscle) and mental work (brain). Physical and mental activity will lead to the emergence of workload. The workload is the difference between a worker's ability and job demands (Meshkati \& Hancock, 1988). Should the ability of the workers is higher than the demands of the work it will cause a sense of boredom and vice versa, if the ability of workers is lower than the demands of the work it will cause excessive fatigue that causes stress work on employees and cause frequent occurrence of human error that led to work accidents.

This study focused on measuring the mental workload of drivers on the street environment, driving tasks, and drivers personality. The method used in this study is NASA-TLX. This method can analyse the mental workload of a driver. The basic reason for selecting the NASA TLX method is the ease of measurement implementation [7]. The NASA - TLX method was developed by Sandra G. of the NASA research center and Lowell E. Staveland of the San Jose State University in 1981. NASA TLX is generally composed of 2 main parts of rating and weighting. The rating was obtained from the respondents on what was felt during the completion of the driving assignment with a rating scale of 0 to 100 . This rating factor was developed based on the emergence of subjective measurement needs consisting of 6 scales: Mental demand (MD), Physical demand (PD), Temporal demand (TD), Performance (P), Frustration (F), Effort (EF). Weighting is calculated on the basis of pairing options deemed most relevant to the workload experienced. Weighting is calculated by the tally of 15 pairwise combination options of the mental load factor. Weighting had values between 0 and 5. Overall workload score (OWS) is calculated by multiplying the rating by weighting the subjective measuring factor on each respondent.

This research was developed using field experiment utilizing similar car facility. Subjective mental workload measurement process was conducted in several streets at Malang and its surrounding city, in East Java, Indonesia. The roads involved were highway, rural road, city road/market during busy working hours. The driver after passing the predetermined path was to immediately fill out the NASA TLX questionnaire which corresponds to what is perceived from the 6-dimensional measurement assessment. There are 26 driver samples performed by driving performance measurement with varied driving period experience. Respondent drivers' age was 21 years old on average, with an average driving experience of 4.08 years. Based on Ambarwati et al [8], drivers in Indonesia experiencing a number of accidents were teenagers aged between 18-24 years old. Questionnaires were given to 26 drivers after passing through the streets as much as 3 repetitions targeted such as market/city road, rural road, and highway.

Steps taken in measuring mental workload of drivers utilizing NASA TLX methods which passed through various conditions and street complexity can be described as follows:

1. Preparation of data collection equipment: questionnaire, camera, etc.

2. Set up experiments in test vehicles

3. Experiment implementation

4. Weighting questionnaire result

5. Rating.

6. OWS value calculation

7. Workload assessment categorization

8. Analysis and discussion

\section{Results and Analysis}

Figure 1 illustrates the situation and the complexity of driving across the city road, rural road and highway in Malang, Indonesia. The figure exhibits hazards and constraints encountered by drivers passing through the city such as pedestrian crossing the street, parking vehicles, etc.. On the other hand, rural road tend to have simple road network condition, more drivers interact with other vehicles. As for highway riders concentrate more on their respective vehicles and road structure. It involves streets' ascent, descent, turn or bump.

Data on mental workload using NASA-TLX method used six indicators to determine mental workload experienced by the driver. Indicators are Mental Demand (MD), Physical Demand (PD), Temporal Demand (TD), Performance (P), Frustration level (F), Effort (EF). On the other hand, weighting stage presents 15 pairs of mental load indicator, to be filled by the respondent (driver) by circling one of the indicator pairs which they thought to be more dominant or important on driving activity. The weighting factor of mental workload constraints on city road examination result is exhibited in Table 1 with average MD = 3.62, $\mathrm{PD}=1.31, \mathrm{TD}=1.81, \mathrm{P}=1.50, \mathrm{~F}=3.92$, and $\mathrm{EF}=2.85$. Based on questionnaire records on rural road, mental load factor value obtained were as follows: $\mathrm{MD}=3.35, \mathrm{PD}=1.81, \mathrm{TD}=1.88, \mathrm{P}=1.73, \mathrm{~F}=3.46$, and $\mathrm{EF}=2.77$. Value obtained for highway section was: $\mathrm{MD}=3.19, \mathrm{PD}=1.54, \mathrm{TD}=2.35, \mathrm{P}=2.88, \mathrm{~F}=2.50$, and $\mathrm{EF}=2.77$. Based on research data, driving in city road requires more and dominant mental effort compared to physical needs. It also has a high level of stress which is supported by the value of low job satisfaction in completing the task. 

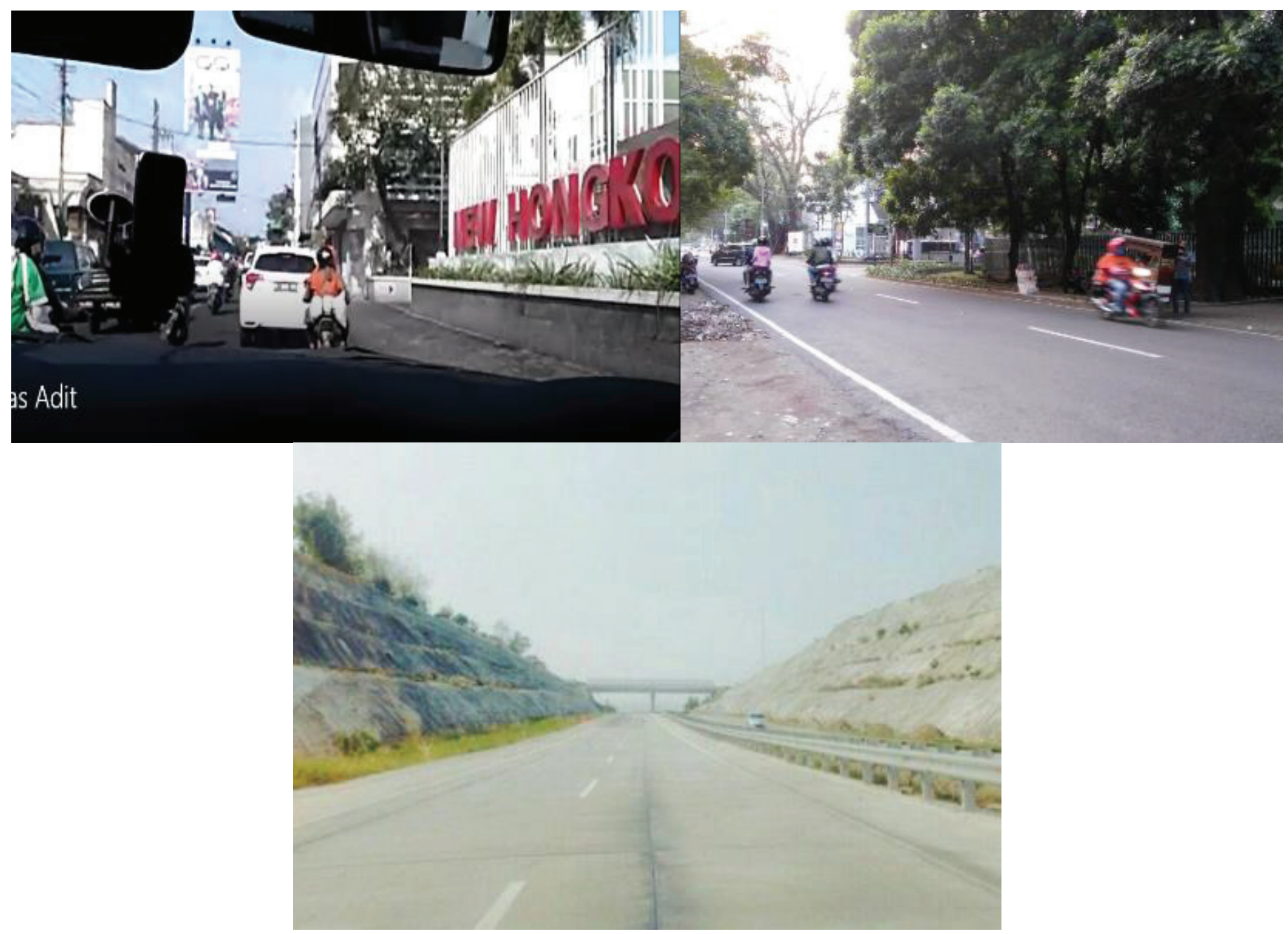

Figure 1. Description of the driving situation on three different road conditions: a. City Road, b. Rural Road, C. Highway.

Table 1. Weighted Data of NASA TLX Questionnaire on city road

\begin{tabular}{|c|c|c|c|c|c|c|c|}
\hline \multirow{2}{*}{ Driver } & \multicolumn{6}{|c|}{ Indicator } & \multirow{2}{*}{ Total Score } \\
\hline & MD & PD & TD & $\mathbf{P}$ & $\bar{F}$ & EF & \\
\hline Driver 1 & 4 & 2 & 0 & 1 & 5 & 3 & 15 \\
\hline Driver 2 & 3 & 1 & 4 & 0 & 5 & 2 & 15 \\
\hline- & - & - & - & - & - & - & - \\
\hline Driver 25 & 3 & 2 & 2 & 2 & 3 & 3 & 15 \\
\hline Driver 26 & 4 & 1 & 1 & 2 & 3 & 4 & 15 \\
\hline
\end{tabular}

Rating stage follows weighting stage. At this stage, a rating of 1-100 scale is given for each indicator according to the circumstances experienced by the driver. Rating distribution comparison result for all indicators on each track type is exhibited in Figure 2 below. The figure exhibited driver driving through city road has the greatest mental burden. They possess the highest frustration, effort, and mental demand. On the other hand, performance has the lowest value. Drivers passing through the rural roads tend to give a good performance, despite experiencing a high level of frustration and hard effort. The overall highway has a positive impact on drivers which exhibits low levels of frustration and physical demand but results in high satisfaction of driving achievement.

The following step is to calculate Overall Workload Score (OWS) which aims to obtain the value of mental workload on each indicator. The category of workload assessment consists of three levels: low with the interval scale of $0-9$, medium for interval scale is 10 - 29, slightly high at interval scale of $30-49$, high at interval scale 50 - 79 and very high with interval scale of 80 - 100 . Below is an example of a calculation recapitulation of OWS driver who drives on city road.

$$
\begin{aligned}
& =((80 \times 4)+(75 \times 2)+(65 \times 0)+(85 \times 1)+(85 \times 5)+(90 \times 3)) / 15 \\
& =(320+150+85+425+270) / 15
\end{aligned}
$$




$$
=1250 / 15
$$

$=88.33$

Based on calculation results above, driver mental workload in a subjective measurement can be classified in "very high" category. Overall it can be classified that the drivers traveling on city road and rural road will experience a mental burden of $3.85 \%$ in "very high" category and $96.15 \%$ in "high" category. Drivers traveling in highway are 100\% categorized in "high" workload. Car technology, proper driving training and improving driver's own health level.

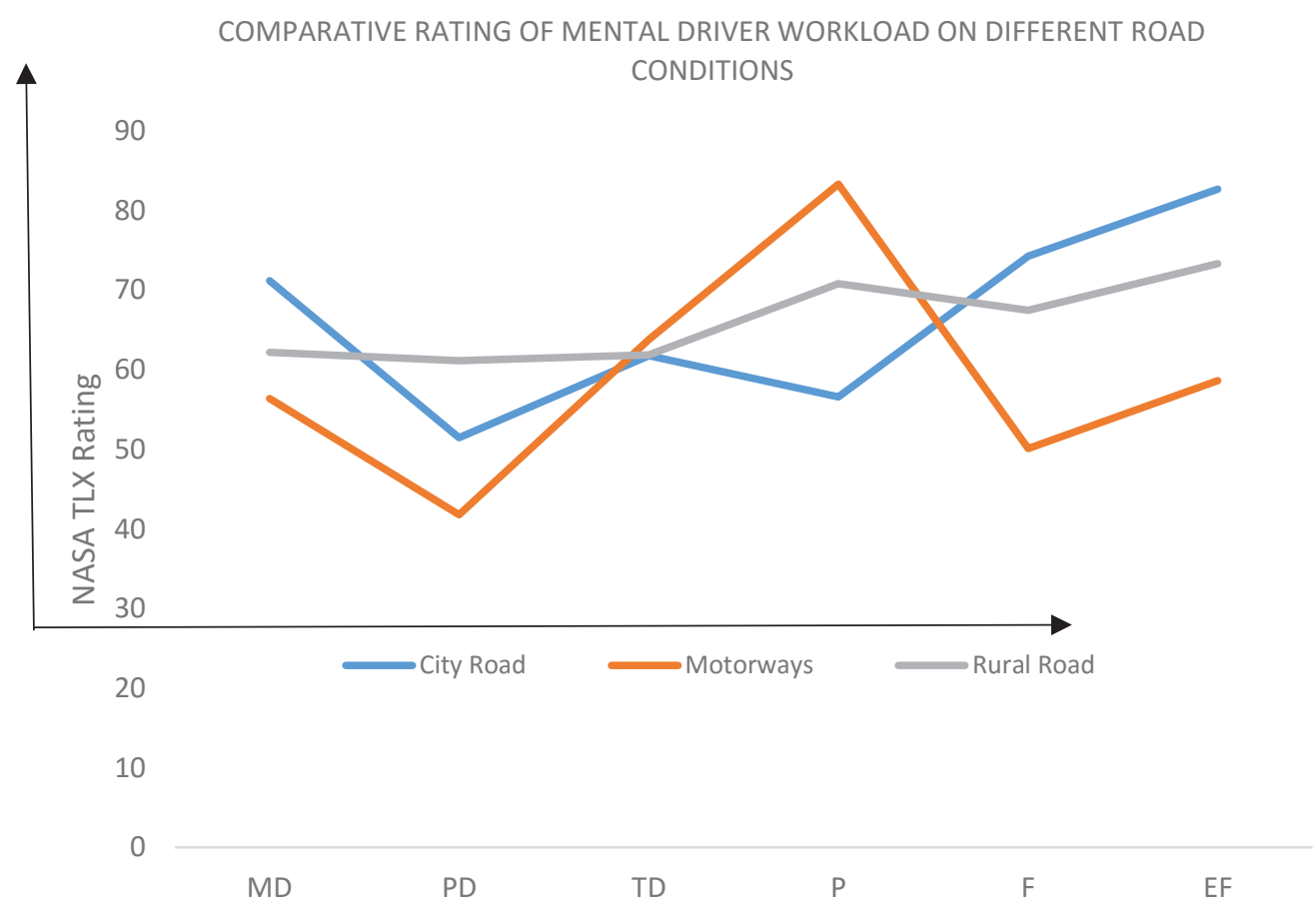

Figure 2. Rating comparison on each NASA TLX indicators in different road conditions

\section{Conclusions}

This research has successfully demonstrated how NASA TLX can be utilized to measure subjective mental workload on drivers crossing different types of streets (city road, rural road, highway) around Malang City, Indonesia. Based on measurement results, it was exhibited that almost every respondent have a mental workload in "high" category. Mental demand frustration factor becomes the main cause of high mental work load, then followed by performing hard effort to reach their purpose. Physical needs have not become a serious consideration compared to other factors to achieve driver satisfaction. Therefore special handling based on driver experience is required. It is recommended to improve infrastructure, transport regulations, and innovation of safe and comfortable driving technologies.

\section{Acknowledgements}

The researcher expresses their gratitude to Ministry of National Education of the Republic of Indonesia for supporting this paper. The authors are also grateful to the Work Design and Ergonomics Laboratory, Industrial Engineering Department, University of Brawijaya, Malang, Indonesia for their extraordinary support.

\section{References}

1. Fastenmeier, W. \& Gstalter, H., SC, 45, 952-979 (2007).

2. Fuller R., AAP, 37 461-472 (2005).

3. Schlegel, R.E, Automotive Ergonomics, Taylor \& Francis, London (1993).

4. Bhise, V. D, Ergonomics in the Automotive Design Process, Boca Raton, CRC Press, (2012).

5. Verwey, W. B. \& ZaideL, D. M., AAP 31(3), 199-211 (1999).

6. Shih-Nan Lu, et al., TJSE, 13 (4), pp. 395404 (2010). 
7. Alex Cao et al., BRM, 41(1), 113 -117 (2009).

8. Ambarwati L. et al., JRS, 4(2), (2010). 